\title{
Dynamic stability control in younger and older adults during stair descent.
}

Citation for published version (APA):

Bosse, I., Oberlander, K. D., Savelberg, H. H., Meijer, K., Bruggemann, G. P., \& Karamanidis, K. (2012). Dynamic stability control in younger and older adults during stair descent. Human Movement Science, 31(6), 1560-1570. https://doi.org/10.1016/j.humov.2012.05.003

Document status and date:

Published: 01/01/2012

DOI:

10.1016/j.humov.2012.05.003

Document Version:

Publisher's PDF, also known as Version of record

Document license:

Taverne

Please check the document version of this publication:

- A submitted manuscript is the version of the article upon submission and before peer-review. There can be important differences between the submitted version and the official published version of record.

People interested in the research are advised to contact the author for the final version of the publication, or visit the DOI to the publisher's website.

- The final author version and the galley proof are versions of the publication after peer review.

- The final published version features the final layout of the paper including the volume, issue and page numbers.

Link to publication

\footnotetext{
General rights rights.

- You may freely distribute the URL identifying the publication in the public portal. please follow below link for the End User Agreement:

www.umlib.nl/taverne-license

Take down policy

If you believe that this document breaches copyright please contact us at:

repository@maastrichtuniversity.nl

providing details and we will investigate your claim.
}

Copyright and moral rights for the publications made accessible in the public portal are retained by the authors and/or other copyright owners and it is a condition of accessing publications that users recognise and abide by the legal requirements associated with these

- Users may download and print one copy of any publication from the public portal for the purpose of private study or research.

- You may not further distribute the material or use it for any profit-making activity or commercial gain

If the publication is distributed under the terms of Article $25 \mathrm{fa}$ of the Dutch Copyright Act, indicated by the "Taverne" license above, 


\title{
Dynamic stability control in younger and older adults during stair descent
}

\author{
Iris Bosse ${ }^{\mathrm{a}}$, Kai Daniel Oberländer ${ }^{\mathrm{b}}$, Hans Hubert Savelberg ${ }^{\mathrm{a}}$, \\ Kenneth Meijer ${ }^{\mathrm{a}}$, Gert-Peter Brüggemann ${ }^{\mathrm{b}}$, Kiros Karamanidis ${ }^{\mathrm{b}, *}$ \\ a Department of Human Movement Sciences, Faculty of Health, Medicine and Life Sciences, Maastricht University, P.O. Box 616, \\ 6200 MD Maastricht, The Netherlands \\ ${ }^{\mathrm{b}}$ German Sport University Cologne, Institute of Biomechanics and Orthopedics, Am Sportpark Müngersdorf 6, 50933 Cologne, Germany
}

\section{A R T I C L E I N F O}

\section{Article history:}

Available online 31 July 2012

PsycINFO classification:

2860

Keywords:

Aging

Gait

Dynamic stability

Falls

\begin{abstract}
A B S T R A C T
The purpose of this study was to examine dynamic stability control in older and younger adults while descending stairs. Thirteen older (aged 64-77 years) and 13 younger (aged 22-29 years) adults descended a staircase at their preferred speed. A motion capture system and three force plates were used to determine locomotion mechanics. Dynamic stability was investigated by using the margin of stability, calculated as the instantaneous difference between anterior boundary of the base of support and extrapolated centre of mass. At the initiation of the single support phase, older adults demonstrated a more negative $(p<.05)$ margin of stability value. The component responsible for the lower margin of stability in the elderly was the higher velocity of the centre of mass $(p<.05)$. Before the initiation of the single support phase, the older adults showed a lower $(p<.05)$ ankle and knee joint angular impulse compared to the younger ones. We found a significant correlation $(r=.729, p<.05)$ between centre of mass velocity and joint angular impulse. These results indicate that older adults are at greater risk of falls while descending stairs potentially due to a reduced ability to generate adequate leg-extensor muscular output to safely control the motion of the body's centre of mass while stepping down. (c) 2012 Elsevier B.V. All rights reserved.
\end{abstract}

\footnotetext{
* Corresponding author. Tel.: +49 (0)221 4982 5680; fax: +49 (0)221 4971598.

E-mail addresses: irisbosse@gmail.com (I. Bosse), k.Oberlaender@dshs-koeln.de (K.D. Oberländer), hans.savelberg@maastrichtuniversity.nl (H.H. Savelberg), kenneth.meijer@maastrichtuniversity.nl (K. Meijer), Brueggemann@dshs-koeln.de (G.-P. Brüggemann), Karamanidis@dshs-koeln.de (K. Karamanidis).
} 


\section{Introduction}

Falls are the leading cause of disability and loss of independence in the elderly population (Tinetti \& Williams, 1997). Epidemiological studies have shown that about one-third of those aged 65 years or older report at least one fall per year (Centers for Disease Control \& Prevention (CDC), 2008; Tinetti, Speechley, \& Ginter, 1988). The likelihood of falling increases considerably with advancing age and most falls occur during daily locomotion activities (Centers for Disease Control \& Prevention (CDC), 2008). In particular, stair negotiation is among the most demanding and hazardous types of locomotion tasks for the elderly (Startzell, Owens, Mulfinger, \& Cavanagh, 2000). About 10\% of fall-related fatalities were reported to occur during stair descent (Startzell et al., 2000). Fall-related injuries can result in death, disability and nursing home admission, and increase the costs of the health care system (Stevens, Corso, Finkelstein, \& Miller, 2006).

Stair descent is clearly distinguishable from other activities, since the demands that stair descent places on the musculoskeletal system are compounded by the need to safely control the body's centre of mass (CoM) while stepping down (Lee \& Chou, 2007; Mian, Narici, Minetti, \& Baltzopoulos, 2007; Zachazewski, Riley, \& Krebs, 1993). Using an instrumented knee implant, Heinlein et al. (2009) showed that the joint moments at the knee are considerably higher during stair descent than during other locomotion activities such as level walking. As a consequence, the ability to negotiate stairs safely is dependent on adequate lower limb muscle strength (Karamanidis \& Arampatzis, 2011; Reeves, Spanjaard, Mohagheghi, Baltzopoulos, \& Maganaris, 2008; Salem, Wang, Young, Marion, \& Greendale, 2000). In literature it is well established that the ageing process is associated with a gradual decline in muscle strength (D'Antona et al., 2003; Frontera et al., 2000) and tendon stiffness (Karamanidis \& Arampatzis, 2005; Mademli \& Arampatzis, 2008), leading to decreased leg-extensor muscle-tendon unit capacities in the elderly. Due to a higher muscle force potential of the trailing leg compared to the leading leg, older adults use their trailing leg before the initiation of the double support phase more while descending stairs compared to younger participants (Karamanidis \& Arampatzis, 2011). Furthermore, older adults make greater use of their stronger knee-extensor muscles than of their more distal ankle plantarflexors while descending stairs in comparison to younger adults (Karamanidis \& Arampatzis, 2011; Reeves et al., 2008). The results are in agreement with the literature reports (DeVita \& Hortobagyi, 2000; Savelberg, Verdijk, Willems, \& Meijer, 2007) showing an age-related redistribution of the joint moments at the ankle, knee and hip in a distal to proximal fashion while level walking.

The findings mentioned above demonstrate that older adults descend stairs with an altered control strategy possibly aimed at adjusting task demand to their reduced leg-extensor muscle-tendon unit capacities. The reported age-related compensatory adjustments in the stair descent control strategy may affect the position and velocity of the CoM while stepping down. This assumption is based on the observation that trunk angular acceleration is affected by lower extremity joint moment output during locomotion (Nott, Zajac, Neptune, \& Kautz, 2010). Lee and Chou (2007) recently reported an increase in the sagittal plane inclination angle between the CoM and the centre of pressure (CoP) in older compared to younger adults during stair descent, reflecting a lower ability to regulate body sway while stepping down among the elderly. In addition, older adults spent more time looking at the next steps than younger subjects did before stepping onto the stairs (Zietz \& Hollands, 2009). This altered gaze behavior for the elderly may be accomplished by a more flexed trunk, which in turn can cause an anterior shift of the CoM in comparison to younger adults while descending stairs.

Dynamic stability during locomotion can be quantified by the margin of stability (Hof, Gazendam, \& Sinke, 2005). This reflects the instantaneous difference between the base of support and the extrapolated CoM (ascertained by the position and velocity of the CoM). During the double support phase of stair descent, the base of support confines the area between the steps of the staircase of both feet. At the single support phase, the base of support is limited to the tread depth of a step so the anterior boundary of the base of support can be defined by the edge of the step. This is because the forefoot of an adult subject usually exceeds the edge of the step while descending stairs. This clearly distinguishes stair descent from other daily locomotion activities. Since foot placement and step length are restricted by the geometry of the stairs, dynamic stability in the anterior-posterior direction 
mainly depends on the position and velocity of the CoM. According to this, an unstable body configuration in the anterior direction apparently occurs if the position of the extrapolated CoM exceeds the edge of the step, potentially explaining the high risk of falling while descending stairs among the elderly population.

Based on the equation defined by Hof et al. (2005), dynamic stability control has been described in detail during suddenly induced forward falls in the elderly (Arampatzis, Karamanidis, \& Mademli, 2008; Karamanidis, Arampatzis, \& Mademli, 2008; Arampatzis, \& Karamanidis, 2008). Briefly, after a sudden perturbation, unstable older individuals showed a lesser ability to rapidly increase their base of support in relation to the extrapolated CoM than stable participants, leading to an increased risk of falls for the unstable older adults (Arampatzis et al., 2008). However, to our knowledge there is no clear information about dynamic stability and its components in younger and older adults while descending stairs using the concept of the extrapolated CoM provided by Hof et al. (2005). Given the altered stair descent control strategy in the elderly, we hypothesised that older adults will show a more anterior position of the extrapolated CoM relative to the edge of the step compared to younger adults, leading to a reduced dynamic stability while descending stairs. The purpose of this study was, therefore, to examine the components of dynamic stability in a group of older and younger adults while descending stairs at their preferred speed and, thus, to determine whether the elderly are at greater risk of falls during stair descent compared to younger adults.

\section{Materials and methods}

\subsection{Experimental design}

Thirteen healthy older adults (seven males, six females; mean $\pm S D$ age: $69 \pm 4$ yrs) and 13 healthy young adults (six males, seven females; age: $25 \pm 2$ yrs), all physically active and with similar anthropometrical characteristics with respect to body mass (younger adults: $73 \pm 9 \mathrm{~kg}$; older adults: $71 \pm 6 \mathrm{~kg}$ ) and body height (younger adults: $171 \pm 7 \mathrm{~cm}$; older adults: $171 \pm 5 \mathrm{~cm}$ ) participated in the study. All subjects were screened to exclude significant neuromusculoskeletal impairments (e.g., complex trauma, multiple sclerosis, cerebral palsy and amputation of the lower extremity) and any lower extremity degenerative joint disease. The experimental procedures for the study were explained to the subjects and written informed consent was obtained prior to the testing, following the guidelines of the local ethical board.

The participants descended a two-step staircase (riser height of each step: $17 \mathrm{~cm}$; tread depth of each step: $30 \mathrm{~cm}$; tread width of each step: $39 \mathrm{~cm}$ ) in a step-over manner with their arms in their natural position. The size and geometry of the steps comply with the National Standards of Germany (German Industry Norms). Participants were requested to walk at their preferred speed while wearing their own flat casual street shoes in order to achieve natural movement patterns. Subjects were not allowed to use any high heels, boots or special athletic shoes such as basketball shoes. No handrails were available. To accommodate themselves to the conditions, all participants were allowed to perform as many practice trials as required. For each subject, three valid trials were collected for the analysis.

Three force plates (Kistler, Winterthur, Switzerland, $60 \times 40 \mathrm{~cm}^{2}, 1080 \mathrm{~Hz}$ ) were used to assess the ground reaction forces. The force plates were embedded on the ground and placed under the steps. In order to measure the forces of each force plate independently, each of the steps was separately secured, offering a small distance between them. Kinematics were obtained using a Vicon Nexus capture system (Vicon Motion Systems, Oxford, UK) with 13 infrared cameras operating at a sampling rate of $120 \mathrm{~Hz}$.

Participants always started in a double support standing position. Stair descent was initiated with the left leg (contra lateral leg) on the level floor, followed by a step down onto the staircase with the right leg (analysed leg). The left leg continued the step sequence while making contact with the second step of the staircase before walking on level floor. The first step down with the right leg onto the staircase was intended for kinematic and kinetic analysis. The other force plates served to identify the gait cycle events (touch down and take off of the right and left leg). For each trial five events (time 
points) were identified (Fig. 1): (1) take off of the analysed right leg, (2) touch down of the analysed right leg, (3) take off of the contra-lateral left leg, (4) touch down of the contra-lateral left leg and (5) take off of the analysed right leg. Accordingly, the swing phase and stance phase of the analysed right leg were defined from event 1 to 2 and from 2 to 5 , respectively. The double support phase of the analysed right leg was defined from event 2 to 3 (Fig. 1). The gait cycle events 2, 4 and 5 were identified by using the ground reaction force with a threshold level of $20 \mathrm{~N}$. For events 1 and 3 there was no ground reaction force available and, therefore, those two events were determined by the trajectories of the foot markers. In the following text event 2 is defined as initiation of the double support phase and event 3 as the initiation of the single support phase (Fig. 1).

\subsection{Joint kinematic and kinetic analysis}

To track complete body kinematics while descending stairs, a multisegment system defined by 39 reflective markers (diameter $14 \mathrm{~mm}$ ) was used and classified into a soft tissue deformation optimized full body model of 13 segments (feet, shanks, thighs, forearms, upper arms, pelvis, torso and head; for exact description of the method please see Oberländer, Brüggemann, Höher, \& Karamanidis, 2012).

Stair descent mechanics of the subjects were described with reference to a neutral upright position of each participant realized before measurement. The neutral upright position was determined while the subject was standing erect (tibia perpendicular to the foot, femur in line with the tibia and trunk in an erect position), facing directly forward, legs slightly apart, feet pointed forward, shoulders $90^{\circ}$ abducted and arms straightened sideways in parallel to the ground with palms facing downward (Oberländer et al., 2012). To remediate soft tissue artefacts during stair descent, the marker positions of the lower extremity and of the head and pelvis were optimized with respect to the neutral upright position by a singular value decomposition algorithm provided by Söderkvist and Wedin (1993).

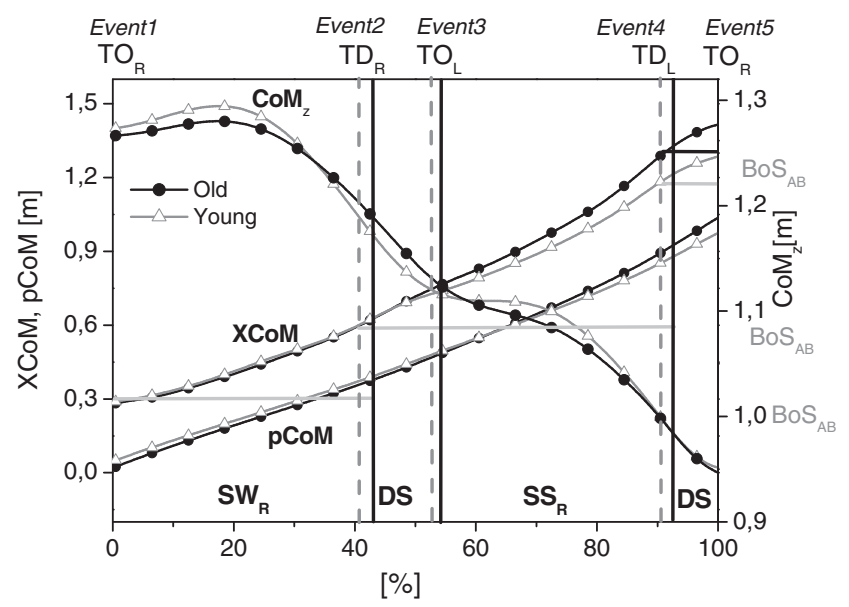

Fig. 1. Anterior boundary of the base of support $\left(\mathrm{BoS}_{\mathrm{AB}}\right)$, vertical oscillation of the centre of mass $(\mathrm{CoMz})$, anterior-posterior component of the vertical projection of the CoM to the ground ( $\mathrm{PCoM})$ and the extrapolated CoM (XCoM; ascertained by the position and velocity of the CoM) for the older $(n=13)$ and the younger $(n=13)$ adults while descending stairs (mean). The $x$ axis was normalized from 0 to $100 \%$ of the stride cycle. The vertical dashed and solid lines represent the instants of touch down (TD) and take off (TO) of the right and left foot for the younger and older adults, respectively. $\mathrm{SW}_{\mathrm{R}}$ : swing phase of the analysed right leg; DS: double support phase; $\mathrm{SS}_{\mathrm{R}}$ : single support phase of the analysed right leg. The margin of stability reflects the instantaneous difference between the $\mathrm{BoS}_{\mathrm{AB}}$ and the $\mathrm{XCoM}$. The $\mathrm{BoS}_{\mathrm{AB}}$ was defined by the edge of the step because the position of the centre of pressure under the foot is limited by the geometry of the stair. This was proved and verified for each analysed trial by taking into consideration the horizontal component of the projection of the metatarsal head III marker (right leg) in relation to the edge of the step. For the staircase-to-floor transition (event 4 to 5 ), the trajectories of the foot markers were used to define the $\operatorname{BoS}_{\mathrm{AB}}$. Please note that the values of the $\mathrm{BoS}_{\mathrm{AB}}$ refer to the left $y$-axis. 
The external joint moments at the ankle and knee joint of the right leg were calculated through inverse dynamics (Hof, 1992). The masses, moments of inertia and CoM position of the various body segments were calculated using the data provided by Zatsiorsky and Seluyanov (1983). Ankle and knee joint angular impulse ( $\mathrm{Nm} \mathrm{s} \mathrm{kg}^{-1}$ ) at the right leg was calculated for the double support phase by the integral of the ankle and knee joint moment over the double support time. In order to determine the braking impulse at the leading leg the integral of the posterior ground reaction force over the time was calculated during the same interval. Ankle and knee joint angles were determined for the right leg at the initiation of the double support phase (event 2: instants of touch down of the right leg) and at the initiation of the single support phase (event 3: instants of take off of the left leg).

\subsection{Dynamic stability analysis}

To quantify dynamic stability while descending stairs we applied the inverted pendulum model defined by Hof et al. (2005). Within this model the margin of stability in the anterior-posterior direction is calculated as the difference between the anterior-posterior boundary of the base of support and the extrapolated CoM (XCoM). Because foot placement and step length, and hence the position of the CoP under the foot, are limited by the geometry of the staircase, the anterior boundary of the base of support was defined by the edge of the stair. This was proved and verified for each analysed trial by taking into consideration the horizontal component of the projection of the metatarsal head III marker of the right leg in relation to the position of the edge of the stair in the global coordinate system. XCoM was defined as follows (Hof et al., 2005; Fig. 1):

$$
\mathrm{XCoM}=\mathrm{pCoM}+\frac{\mathrm{vCoM}}{\sqrt{\mathrm{gl}^{-1}}}
$$

where pCoM denotes the anterior-posterior component of the vertical projection of the CoM to the ground, vCoM the anterior-posterior velocity of the CoM, g the gravity and 1 the distance between $\mathrm{CoM}$ and centre of the ankle joint. Postural stability is warranted if the position of the extrapolated $\mathrm{CoM}$ is within the base of support (positive values of margin of stability) while stability is lost when the extrapolated CoM exceeds the boundary of the base of support (negative values of margin of stability). Margin of stability was determined in the anterior-posterior direction because stair descent falls generally occur in the anterior direction. The validity of the model during functional activities has been demonstrated in previous studies (Arampatzis et al., 2008; Karamanidis et al., 2008; Mademli et al., 2008).

Trunk flexion angle was determined as the sagittal plane projection of the line connecting the midpoint of the trans-acromion line, the midpoint of the trans-iliac crest line and the global vertical axis in relation to the neutral upright position (Asay, Mündermann, \& Andriacchi, 2009). As for the joint kinematics, the components of the dynamic stability and trunk flexion angle were determined at the initiation of the double support phase (event 2: instants of touch down of the right leg) and at the initiation of the single support phase (event 3: instants of take off of the left leg).

\subsection{Statistics}

For the statistical analysis, the mean values from three valid trials were utilized for each subject. A univariate analysis of variance was performed with age (old vs. young) as factor in order to identify differences in the analysed dynamic stability parameters (margin of stability, extrapolated CoM, anterior-posterior component of the vertical projection of the CoM to the ground, anterior-posterior velocity of the CoM and distance between CoM and centre of the ankle joint), joint angles (ankle, knee and trunk), braking impulse (i.e., time integral of the negative anterior-posterior ground reaction force), and ankle and knee joint angular impulse between older and younger adults. Further, we used a Pearson correlation coefficient analysis and a multiple regression analysis with joint angular impulse and the components of the dynamic stability as variables to identify the relationship between joint kinetics and dynamic stability. The level of significance was set for $p$-values less than .05 . All results are presented as means and standard deviation $(S D)$. 


\section{Results}

\subsection{Initiation of the double support phase}

Older participants showed a tendency $(p=.09)$ towards a smaller margin of stability at the initiation of the double support phase compared to younger adults (Table 1). This tended to occur with a higher anterior CoM velocity $(p=.07)$, leading to a more anterior position of the extrapolated CoM $(p=.09)$ for the older adults (Table 1$)$. Further, older adults demonstrated a tendency $(p=.06)$ of a greater trunk flexion angle at this time point of the gait cycle (Table 1). There was no significant age effect on ankle $(p=.26)$ or knee $(p=.22)$ joint angles of the leading leg at the initiation of the double support phase (Table 1 ).

\subsection{Initiation of the single support phase}

At the initiation of the single support phase (at take off of the trailing leg), older adults showed a significantly higher anterior velocity of the CoM $(p=.02)$, a higher extrapolated CoM $(p=.04)$ and hence a lower margin of stability $(p=.04)$ compared to younger participants (Table 1 ). CoM position (anterior-posterior component of the vertical projection of the CoM to the ground and distance

Table 1

Dynamic stability and lower extremity joint kinematics of the leading leg at the initiation of the double support phase and at the initiation of the single support phase for the older $(n=13)$ and younger $(n=13)$ adults while descending stairs $($ mean $\pm S D)$. A negative value of the margin of stability represents a dynamic unstable body configuration while postural stability is warranted when the value of the margin of stability is positive.

\begin{tabular}{lccc}
\hline & Old & Young & $p$-value \\
\hline Initiation of the double support phase & & & \\
Dynamic stability & & & .09 \\
MoS (cm) & $-4.1 \pm 5.6$ & $-0.6 \pm 4.4$ & .09 \\
XCoM (cm) & $63.1 \pm 5.6$ & $59.6 \pm 4.4$ & .40 \\
pCoM (cm) & $37.6 \pm 2.8$ & $36.8 \pm 2.2$ & .07 \\
vCoM (m/s) & $0.86 \pm 0.13$ & $0.77 \pm 0.10$ & .53 \\
l (cm) & $85.2 \pm 4.4$ & $86.2 \pm 3.4$ & .22 \\
Joint kinematics & & & .26 \\
Knee joint angle (deg) & $11.1 \pm 4.5$ & $13.6 \pm 5.7$ & .06 \\
Ankle joint angle (deg) & $23.3 \pm 8.0$ & $26.3 \pm 4.9$ & \\
Trunk flexion angle (deg) & $-12.9 \pm 5.9$ & $-8.9 \pm 4.2$ & \\
Initiation of the single support phase & & & .04 \\
Dynamic stability & & & .04 \\
MoS (cm) & & & .62 \\
XCoM (cm) & & \\
pCoM (cm) & $-18.0 \pm 5.2$ & $-13.6 \pm 4.9$ & .02 \\
vCoM (m/s) & $77.0 \pm 5.2$ & $72.6 \pm 4.9$ & .85 \\
l (cm) & $48.1 \pm 3.0$ & $47.6 \pm 2.2$ & \\
Joint kinematics & $0.99 \pm 0.13$ & $0.85 \pm 0.13$ & \\
Knee joint angle (deg) & $84.4 \pm 4.4$ & $84.1 \pm 3.6$ & .01 \\
Ankle joint angle (deg) & & & .22 \\
Trunk flexion angle (deg) & $20.3 \pm 7.9$ & $29.7 \pm 6.7$ & .13 \\
\hline CoM:centref & $8.6 \pm 5.1$ & \\
\end{tabular}

CoM: centre of mass; MoS: margin of stability; XCoM: extrapolated CoM; pCoM: anterior-posterior component of the vertical projection of the CoM to the ground; vCoM: anterior-posterior velocity of the CoM; l: distance between CoM and centre of the ankle joint. A zero degree of knee and ankle joint angle represents the neutral upright position (tibia perpendicular to the foot, femur in line with the tibia). A positive value is defined as knee flexion and ankle plantarflexion, respectively. Trunk flexion angle was determined as the sagittal plane projection of the line connecting the midpoint of the trans-acromion line, the midpoint of the trans-iliac crest line and the global vertical axis in relation to the neutral upright position. Please note that the anterior boundary of the base of support is not provided in the table because the edge of the step (i.e., base of support) was always in the same position in the global coordinate system.

* Statistically significant differences between older and younger adults $(p<.05)$. 
between CoM and centre of the ankle joint; $p>.61)$ and trunk flexion angle $(p=.13)$ showed no significant differences between older and younger adults. There was a significant age effect $(p<.01)$ on knee joint angle (less flexed joint angle for the elderly) of the leading leg at the initiation of the single support phase (Table 1). As a consequence, knee joint angular changes at the leading leg were significantly lower $(p<.01)$ for the older $\left(9.2 \pm 5.9^{\circ}\right)$ compared to the younger $\left(16.1 \pm 4.2^{\circ}\right)$ adults during the double support phase. During the same phase the braking impulse at the leading leg was significantly $(p=.02)$ lower for the older $\left(2.00 \pm 1.47 \mathrm{Ns} \mathrm{kg}^{-1}\right)$ compared to the younger ones $\left(4.16 \pm 2.89 \mathrm{Ns} \mathrm{kg}^{-1}\right)$. Swing duration (older: $439 \pm 57 \mathrm{~ms}$ vs. younger: $435 \pm 31 \mathrm{~ms} ; p=.81$ ), stance duration ( $588 \pm 77 \mathrm{~ms}$ vs. $640 \pm 71 \mathrm{~ms} ; p=.11$ ), double support duration $(117 \pm 30 \mathrm{~ms}$ vs. $133 \pm 30 \mathrm{~ms} ; p=.17$ ) and single support duration ( $397 \pm 39 \mathrm{~ms}$ vs. $401 \pm 50 \mathrm{~ms} ; p=.81$ ) were not significantly different between older and younger adults.

The joint moment curves at the ankle and knee during the period of ground contact phase (Fig. 2) indicate that the older adults descend stairs with an altered control strategy and generate lower joint moments at the leading compared to the younger ones. Accordingly, the external ankle dorsiflexion $(p=.05)$ and knee flexion ( $p=.01)$ angular impulse of the leading leg calculated over the double support phase (i.e., period before the initiation of the single support phase) were significantly lower for the older compared to the younger adults (Fig. 3). We found that the anterior velocity of the CoM at the initiation of the single support phase was significantly related to the external ankle dorsiflexion $(r=.651, p<.001)$ and knee flexion $(r=-.634, p<.001)$ angular impulse (Fig. 4). The multiple regression analysis (ankle and knee joint angular impulse, and CoM velocity used as variables) showed

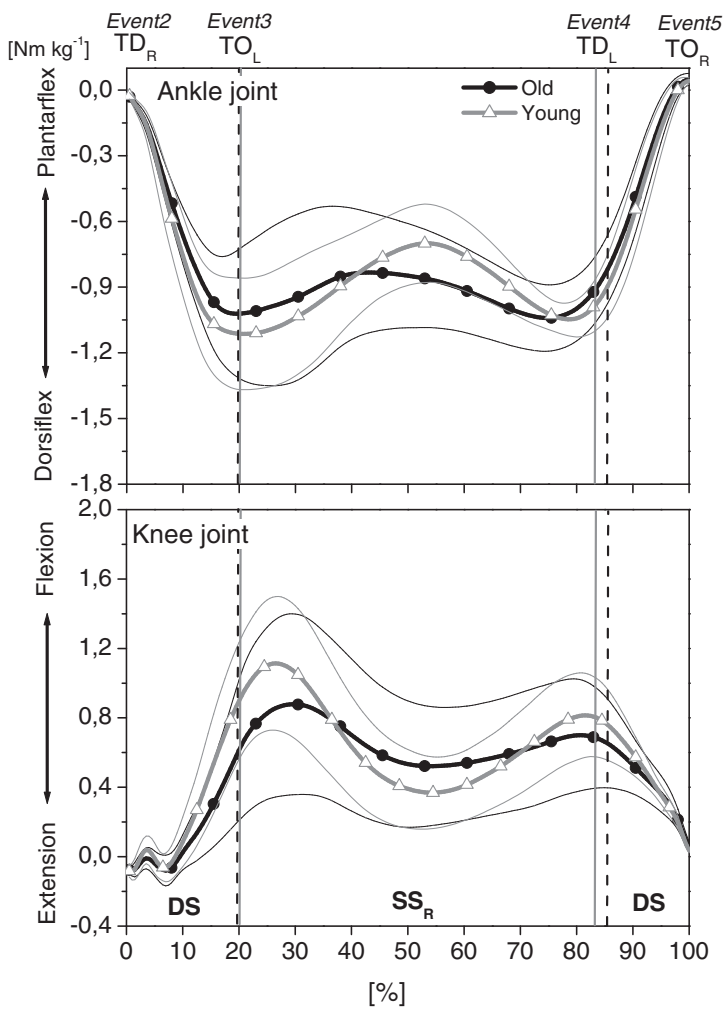

Fig. 2. External joint moments at the ankle and knee joint (right leg) for the older $(n=13)$ and younger ( $n=13)$ adults while descending stairs (mean and $S D$ ). The $x$-axis was normalized from 0 to $100 \%$ of the stance phase. The vertical dashed and solid lines represent the instants of touch down (TD) and take off (TO) of the right and left foot for the older and younger adults, respectively; DS: double support phase; $\mathrm{SS}_{\mathrm{R}}$ : single support phase of the analysed right leg. 


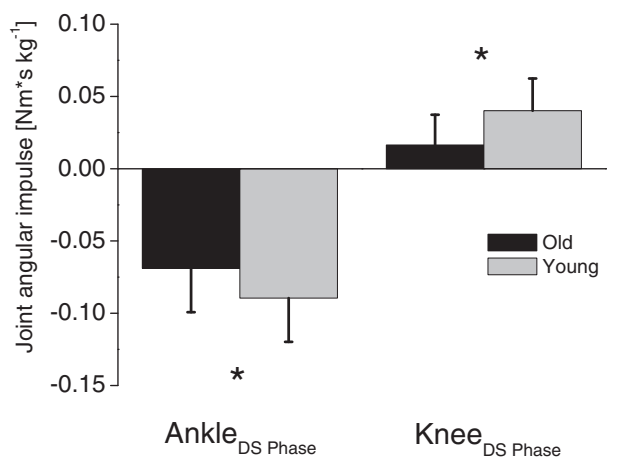

Fig. 3. Magnitude of the external ankle dorsiflexion and knee flexion angular impulse $\left(\mathrm{Nm} \mathrm{s} \mathrm{kg}^{-1}\right)$ at the leading leg during double support phase (DS phase; before the initiation of the single support phase) for the older ( $n=13$ ) and younger ( $n=13$ ) adults while descending stairs (mean and $S D)$. *Statistically significant differences between older and younger adults $(p<.05)$.

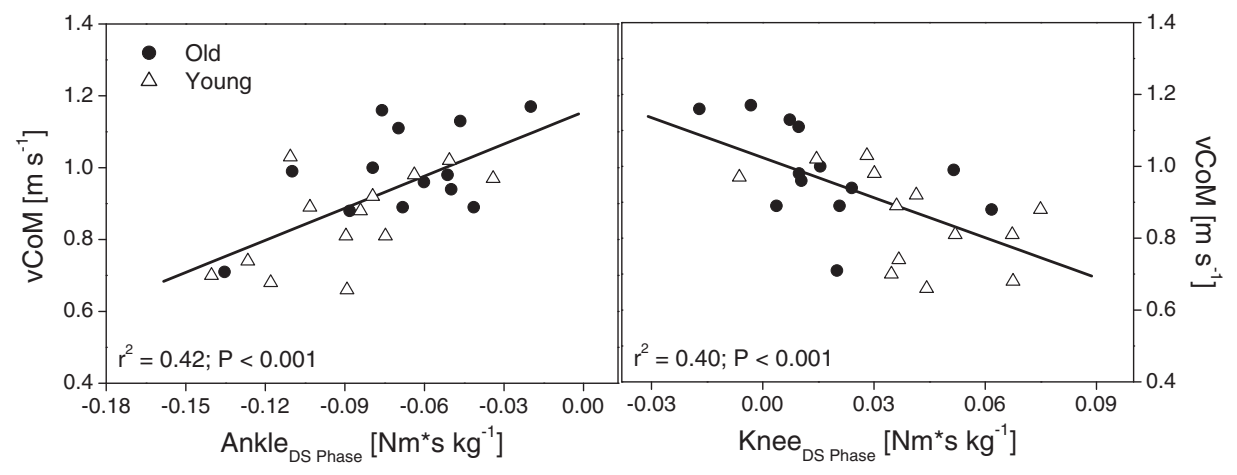

Fig. 4. Relationship between the anterior-posterior velocity of the centre of mass (vCoM) at the initiation of the single support phase and external ankle dorsiflexion and knee flexion angular impulse $\left(\mathrm{Nm} \mathrm{s} \mathrm{kg}^{-1}\right)$ at the leading leg during the double support phase (DS phase; before the initiation of the single support phase) while descending stairs for all examined subjects (old $=13$; young $=13$ ).

a significant relationship $(r=.729, p<.001)$ between leg-extensor angular impulse and anterior velocity of the CoM at the initiation of the single support phase.

\section{Discussion}

This study aimed to examine whether older adults are at greater risk of falls during stair descent compared to younger adults due to a lesser ability to safely control the body's CoM while stepping down. At the initiation of the single support phase (at take off of the trailing leg), older adults had a significantly more negative margin of stability than younger participants, which was on average $33 \%$ lower for the elderly. Considering that the ability to increase the base of support is limited during staircase locomotion, the above findings demonstrate that older adults are at greater risk of falls while descending stairs, confirming our hypothesis.

While descending stairs, the base of support is restricted to the edge of the steps, leading to no differences in this component between age groups. Therefore, the main reason for the lower margin of stability at the initiation of the single support phase was the higher velocity of the CoM leading to a more anterior position of the extrapolated CoM for the elderly population. Before the initiation of the single support phase (i.e., double support phase), the older adults demonstrated a lower posterior 
ground reaction force impulse and lower knee joint angular changes at the leading leg compared to the younger adults. The above results show that the elderly population generated less braking forces by the leading leg and, therefore, the older adults swung forward like a pendulum instead of controlling the movement of the CoM by flexing the knee joint of the leading leg as the younger adults showed before the initiation of the single support phase. This provides evidence that older adults may not be able to effectively reduce their body sway before the initiation of the single support phase of stair descent and, therefore, are at greater risk of falls at this event of the gait cycle.

We found a significant correlation between the velocity of the CoM and ankle and knee joint angular impulse at the leading leg before the initiation of the single support phase (Fig. 4). A multiple regression analysis revealed a significant relationship $(r=.729, p<.001, n=26)$ between velocity of the CoM and leg-extensor angular impulse. Therefore, the results show that the lower joint moment output during the double support phase for the older compared to the younger adults contributed about 53\% to the lesser dynamic stability control at the initiation of the single support phase while stepping down in the elderly. The lower joint moment output at the ankle and knee of the leading leg in older adults are in agreement with earlier studies (Karamanidis \& Arampatzis, 2011; Reeves et al., 2008) and may be associated with a combined effect of reduced leg-extensor muscle-tendon unit capacities (Karamanidis \& Arampatzis, 2005; Mademli et al., 2008) and low force-length potential of the leg-extensor muscles of the leading leg while descending stairs (Chleboun, Harrigal, Odenthal, Shula-Blanchard, \& Steed, 2008; Spanjaard, Reeves, van Dieën, Baltzopoulos, \& Maganaris, 2007). Using ultrasonography, it has recently been shown that at the initiation of the double support phase the fascicle length of the vastus lateralis and gastrocnemius medialis muscle of the leading leg are relatively short and presumably operate at the ascending limb of the force-length relationship (Chleboun et al., 2008; Spanjaard et al., 2007). The above findings clearly diminish older adults' ability to provide the same leg-extensor joint moment output as the younger adults. We may suggest, therefore, that the age-related reduction in ankle plantarflexor and knee-extensor muscle-tendon capacities partly contribute to the increased risk of falls in the elderly while descending stairs.

A tendency $(p=.09)$ of a smaller margin of stability was shown at the initiation of the double support phase (touch down of the leading leg) for the older compared to younger adults while descending stairs. The lower values of the margin of stability in the elderly were associated with a trend $(p=.07)$ of a higher anterior CoM velocity leading to a more anterior position of the extrapolated CoM for older adults compared to younger participants. The age-related changes in the state of the CoM combined with the greater trunk flexion angle by trend $(p=.06)$ can possibly be explained by previous observations, showing that older adults spent more time looking at future stairs than younger subjects did before stepping onto the stairs (Zietz \& Hollands, 2009). Further research is needed to elaborate the effect of gaze behavior in the context of dynamic stability control during staircase locomotion in the elderly.

A limitation of the present study was that the used staircase consisted of two steps. Such a low number of steps may not have allowed our examined subjects to continuously descend the stairs as normal during daily life situations, where most staircases have a larger number of steps. However, because we used the same methodological design for all examined subjects and our age-related changes in joint kinetics is similar to the findings reported in the literature using a longer staircase (Reeves et al., 2008), this drawback may not have influenced our conclusions. Furthermore, gait velocity is difficult to control while descending stairs and, therefore, was not examined in this study. Since swing and stance duration showed no differences between older and younger subjects while stepping down, a disparity in gait velocity between groups may not have significantly affected the current findings.

One might argue that the inverted pendulum model may not be appropriate to describe how the $\mathrm{CoM}$ is controlled during stair descent because during the single support phase the pendulum length actually shortens. As a consequence the extrapolated CoM will in fact be less forward and all participants (older and younger adults) will show a somewhat greater margin of stability than being calculated. However, the length and angle changes in 1 (distance between CoM and centre of the ankle joint) during the single support phase were on average $5.5 \mathrm{~cm}$ (less than $7 \%$ shortening) and $27^{\circ}$ respectively. Because the actual length of the leg is determined by cosine factors and the cosine is close to one at small angles, the inaccuracy of the inverted pendulum model results in a small imprecision of the results but will not affect the overall conclusions of the study. Following the low length changes 
in 1 , the vertical motion of the CoM provides clear evidence of a pendulum mechanism during stair descent (Fig. 1). Moreover, using the CoP-CoM separation as a stability index revealed statistically significant $(p=.01)$ lower values for the older adults compared to the younger ones at the initiation of the single support phase (older: $1.3 \pm 2.7 \mathrm{~cm}$; younger: $4.0 \pm 2.2 \mathrm{~cm}$; a positive value indicates that the $\mathrm{CoM}$ is posterior to the $\mathrm{CoP}$ ). The above finding, when combined with the higher anterior CoM velocity for the elderly, clearly supports our conclusions, i.e., reduced dynamic stability control for the elderly.

It is important to note that all examined older and younger adults showed a negative margin of stability at the initiation of the single support phase. From a mechanical perspective, a negative margin of stability while stepping down is necessary and energetically efficient for transitioning to the next step (Kuo, Donelan, \& Ruina, 2005). The lower braking impulse at the leading leg and as a consequence the lower margin of stability for older adults (more negative value) at the initiation of the single support phase may, therefore, be interpreted to mean that older adults descend stairs energetically more efficiently than younger ones. In that case, we may suggest that older adults sacrifice dynamic stability for ease of motion and for transitioning to the next step.

However, the question remains to be answered why the younger adults did not explore the obviously more efficient motor strategy while descending stairs as shown by the older population. Possibly, a trade-off of the more efficient strategy described above is a hard collision with the ground during the following step, while another may be a less elastic bounce supported by the lower vertical oscillation of the CoM among the elderly (Fig. 1). A reduced elastic bounce is a well-known phenomenon in the elderly and seems to be related to a reduction in muscular force in old age and can result in a lower amount of elastic energy, stored during the deceleration of the CoM (Cavagna, Legramandi, \& Peyré-Tartaruga, 2008). Further studies are needed to elaborate on this issue.

In conclusion, both age groups showed a negative margin of stability at the initiation of the single support phase, which is from a mechanical perspective perhaps energetically efficient for transitioning to the next step but leads to a dynamically unstable body configuration. Older adults demonstrated a significantly more negative margin of stability and, thus, a more unstable body position at the point of the gait cycle where they step down compared to younger ones. At the initiation of the single support phase the margin of stability was on average $-18 \mathrm{~cm}$ for older adults, clearly increasing the risk of forward falls during stair descent in the elderly. The component responsible for the lower margin of stability in the elderly was the higher velocity of the CoM leading to a more anterior position of the extrapolated CoM in relation to the edge of the step. This age-related reduced dynamic stability control while stepping down was partly associated with a lower ankle and knee joint moment output in the elderly. We may conclude, therefore, that older adults are at greater risk of falls while descending stairs potentially due to their reduced ability to generate adequate ankle plantarflexion and knee extension muscular forces to safely control the downwards motion of their body. Increasing leg-extensor muscular capacities in the elderly might be a promising approach for exercise-based interventions, resulting in an increased ability to negotiate stairs in a dynamic, stable manner in old age.

\section{Conflict of interest statement}

The authors have no conflicts of interest to report.

\section{Acknowledgments}

The authors would like to thank Martin Küsel-Feldker, Thomas Förster and their teams for the technical assistance and their active support through the entire project.

\section{References}

Arampatzis, A., Karamanidis, K., \& Mademli, L. (2008). Deficits in the way to achieve balance related to mechanisms of dynamic stability control in the elderly. Journal of Biomechanics, 41, 1754-1761.

Asay, J. L., Mündermann, A., \& Andriacchi, T. P. (2009). Adaptive patterns of movement during stair climbing in patients with knee osteoarthritis. Journal of Orthopaedic Research, 27, 325-329.

Cavagna, G. A., Legramandi, M. A., \& Peyré-Tartaruga, L. A. (2008). Old men running: Mechanical work and elastic bounce. Proceedings of the Royal Society Biological Sciences, 22, 411-418. 
Centers for Disease Control and Prevention (CDC) (2008). Self-reported falls and fall-related injuries among persons aged $>$ or $=$ 65 years-United States, 2006. Morbidity and Mortality Weekly Report, 57, 225-229.

Chleboun, G. S., Harrigal, S. T., Odenthal, J. Z., Shula-Blanchard, L. A., \& Steed, J. N. (2008). Vastus lateralis fascicle length changes during stair ascent and descent. Journal of Orthopaedic E Sports Physical Therapy, 38, 624-631.

D’Antona, G., Pellegrino, M. A., Adami, R., Rossi, R., Carlizzi, C. N., Canepari, M., et al (2003). The effect of ageing and immobilization on structure and function of human skeletal muscle fibres. Journal of Physiology, 552, 499-511.

DeVita, P., \& Hortobagyi, T. (2000). Age causes a redistribution of joint torques and powers during gait. Journal of Applied Physiology, 88, 1804-1811.

Frontera, W. R., Suh, D., Krivickas, L. S., Hughes, V. A., Goldstein, R., \& Roubenoff, R. (2000). Skeletal muscle fiber quality in older men and women. American Journal of Physiology - Cell Physiology, 279, 611-618.

Heinlein, B., Kutzner, I., Graichen, F., Bender, A., Rohlmann, A., Halder, A. M., et al (2009). ESB Clinical Biomechanics Award 2008: Complete data of total knee replacement loading for level walking and stair climbing measured in vivo with a follow-up of 6-10 months. Clinical Biomechanics (Bristol, Avon), 24, 315-326.

Hof, A. L. (1992). An explicit expression for the moment in multibody systems. Journal of Biomechanics, 25, 1209-1211.

Hof, A. L., Gazendam, M. G., \& Sinke, W. E. (2005). The condition for dynamic stability. Journal of Biomechanics, 38, 1-8.

Karamanidis, K., \& Arampatzis, A. (2005). Mechanical and morphological properties of different muscle-tendon units in the lower extremity and running mechanics: Effect of aging and physical activity. Journal of Experimental Biology, 208, 3907-3923.

Karamanidis, K., \& Arampatzis, A. (2011). Altered control strategy between leading and trailing leg increases knee adduction moment in the elderly while descending stairs. Journal of Biomechanics, 44, 706-711.

Karamanidis, K., Arampatzis, A., \& Mademli, L. (2008). Age-related deficit in dynamic stability control after forward falls is affected by muscle strength and tendon stiffness. Journal of Electromyography and Kinesiology, 18, 980-989.

Kuo, A. D., Donelan, J. M., \& Ruina, A. (2005). Energetic consequences of walking like an inverted pendulum: Step-to-step transitions. Exercise E' Sport Sciences Reviews, 33, 88-97.

Lee, H., \& Chou, L. (2007). Balance control during stair negotiation in older adults. Journal of Biomechanics, 40, $2530-2536$.

Mademli, L., \& Arampatzis, A. (2008). Mechanical and morphological properties of the triceps surae muscle-tendon unit in old and young adults and their interaction with a submaximal fatiguing contraction. Journal of Electromyography and Kinesiology, 18, 89-98.

Mademli, L., Arampatzis, A., \& Karamanidis, K. (2008). Dynamic stability control in forward falls: Postural corrections after muscle fatigue in young and older adults. European Journal of Applied Physiology, 103, 295-306.

Mian, O. S., Narici, M. V., Minetti, A. E., \& Baltzopoulos, V. (2007). Center of mass motion during stair negotiation in young and older men. Gait and Posture, 26, 463-469.

Nott, C. R., Zajac, F. E., Neptune, R. R., \& Kautz, S. A. (2010). All joint moments significantly contribute to trunk angular acceleration. Journal of Biomechanics, 43, 2648-2652.

Oberländer, K. D., Brüggemann, G.-P., Höher, J., \& Karamanidis, K. (2012). Reduced knee joint moment in ACL deficient patients at a cost of dynamic stability during landing. Journal of Biomechanics, 45, 1387-1392.

Reeves, N. D., Spanjaard, M., Mohagheghi, A. A., Baltzopoulos, V., \& Maganaris, C. N. (2008). The demands of stair descent relative to maximum capacities in elderly and young adults. Journal of Electromyography and Kinesiology, 18, 218-227.

Salem, G., Wang, M., Young, J., Marion, M., \& Greendale, G. (2000). Knee strength and lower -and higher- intensity functional performance in older adults. Journal of Medicine and Science in Sports and Exercise, 32, 1679-1684.

Savelberg, H. H., Verdijk, L. B., Willems, P. J., \& Meijer, K. (2007). The robustness of age-related gait adaptations: Can running counterbalance the consequences of ageing? Gait and Posture, 25, 259-266.

Söderkvist, I., \& Wedin, P. A. (1993). Determining the movements of the skeleton using well-configured markers. Journal of Biomechanics, 26, 1473-1477.

Spanjaard, M., Reeves, N. D., van Dieën, J. H., Baltzopoulos, V., \& Maganaris, C. N. (2007). Gastrocnemius muscle fascicle behavior during stair negotiation in humans. Journal of Applied Physiology, 102, 1618-1623.

Startzell, J. K., Owens, D. A., Mulfinger, L. M., \& Cavanagh, P. R. (2000). Stairnegotiation in older people: A review. Journal of the American Geriatrics Society, 48, 567-580.

Stevens, J. A., Corso, P. S., Finkelstein, E. A., \& Miller, T. R. (2006). The costs of fatal and non-fatal falls among older adults. Injury Prevention, 12, 290-295.

Tinetti, M. E., Speechley, M., \& Ginter, S. F. (1988). Risk factors for falls among elderly persons living in the community. New England Journal of Medicine, 319, 1701-1707.

Tinetti, M. E., \& Williams, C. S. (1997). Falls, injuries due to falls, and the risk of admission to a nursing home. New England Journal of Medicine, 337, 1279-1284.

Zachazewski, J. E., Riley, P. O., \& Krebs, D. E. (1993). Biomechanical analysis of body mass transfer during stair ascent and descent of healthy subjects. Journal of Rehabilitation Research and Development, 30, 412-422.

Zatsiorsky, V. M., \& Selujanov, V. N. (1983). The mass and inertia characteristics of the main segments of the human body. In H. Matsui \& K. Kabayashi (Eds.), Biomechanics VIII-B (pp. 1152-1159). Champaign, IL: Human Kinetics.

Zietz, D., \& Hollands, M. (2009). Gaze behavior of young and older adults during stair walking. Journal of Motor Behavior, 41, 357-365. 